\title{
Malawi auf dem Weg zur Demokratie? Das Referendum und seine Folgen
}

\author{
Von Heiko Meinhardt 1
}

\section{Einleitung}

Der "wind of change", der seit nunmehr vier Jahren den afrikanischen Kontinent erfaßt hat, hat die meisten autoritären Regime hinweggef egt oder zumindest erschüttert. Waren Ende 1988 noch mit Ausnahme von Mauritius, Botswana, Senegal, Gambia und Zimbabwe alle Regime in Afrika südlich der Sahara autoritär verfaßte Einparteienstaaten, sieht die politische Landschaft heute (Ende 1993) schon sehr viel differenzierter aus. Einer Analyse von Africa Demos folgend, werden (Stand August 1993) 15 Staaten als demokratisch, vier als gelenkte Demokratien und beachtliche 22 Regime als sich im demokratischen Transitionsprozeß befindlich charakterisiert. Drei Regime (Sudan, Swaziland und Zaire) werden als autoritär eingestuft. In Angola, Liberia und Somalia ist infolge von Bürgerkriegen die staatliche Ordnung zusammengebrochen. 2 Dieses Bild macht deutlich, da $B$ in fast allen Staaten politische Veränderungen zu beobachten sind, wemn auch von ganz unterschiedlicher Intensität und Orientierung. In einigen Staaten wurde ein demokratischer Regierungswechsel durch Wahlen vollzogen (Benin, Zambia, Kapverde etc.), in anderen konnte sich das vormals autoritäre Regime in freien Wahlen behaupten (z.B. Kenya, Côte d'Ivoire). Diese relativ übersichtliche und klare Klassifizierung der afrikanischen Regime sollte nicht darüber hinwegtäuschen, daß die jungen Demokratien überaus fragil sind. Ein aktuelles Beispiel dafür ist Burundi. Dort wurde im Juni 1993 in freien und fairen Wahlen eine demokratische Regierung ins Amt gewählt. Nach nur vier Monaten im Amt wurde die gewählte Regierung von Teilen des Militärs gestürzt und der Präsident umgebracht. Als Folge davon entflammte ein Bürgerkrieg, der das Demokratieexperiment erst einmal zum Scheitern verurteilt haben dürfte. 3 Auch in Angola führte die Wahlniederlage von Jonas Savimbi, die er nicht akzeptieren wollte, Ende 1992 zu einer Neuauflage des Bürgerkrieges.

1 Der Autor war als intemationaler Wahlbeobachter während des Referendums in Malawi tätig.

2 Als demokratisch werden Systeme definiert, die einen breiten Wettbewerb organisierter Interessen, politische Partizipationsmöglichkeiten der Bevölkerung, regelmäßige freie und faire Wahlen und Wahrung der Bürger- und Menschenrechte aufweisen. In "gelenkten" Demokratien sind diese Grundelemente zwar formal in der Verfassung festgeschrieben und werden auch respektiert, in der Praxis schränkt jedoch die dominante Stellung des herrschenden Regimes die Wettbewerbschancen von Individuen, Organisationen, Parlamenten und von der Judikative ein. Vgl.: Africa Demos, 1993, vol.3, 2, S. 19.

3 Vgl.: FAZ, 22.10. und 25.10.1993. 
An diesen Beispielen wird deutlich, daB sich die "MeBbarkeit" von Demokratie nicht auf die bloße Existenz demokratischer Institutionen und kompetitiver Wahlen beschränken darf. Sie sind zwar Voraussetzung für eine pluralistische politische Ordnung, bedürfen aber auch eines entsprechenden Umfeldes. So sollten wichtige politische, soziale und ethnische Gruppen nicht ausgegrenzt werden. Ihre Konfliktbereitschaft wächst oft in dem Umfang, in dem ihr Zugriff auf Staatsressourcen geschmälert wird.4

\section{Die Endphase des autoritäten Regimes von Hastings Banda}

Malawi konnte sich sehr lange dem "wind of change" verschließen und gehörte bis zum Sommer 1993 noch in die Kategorie der autoritär verfaßten Staaten.

Hastings Kamuzu Banda regiert den südostafrikanischen Binnenstaat, der mit einem durchschnittlichen BSP p.c. von jährlich nur US \$ 230 (1991) $\mathbf{z u}$ den ärmsten der Welt zählt, seit seiner Unabhängigkeit von Großbritannien 1964. Mit Hilfe eines effizienten Machtund Repressionsapparates gelang es ihm, den Staat wie sein persönliches Eigentum zu führen, woraus er auch kein Geheimnis machte: "I run this state as if it were my own property - I don't let Ministers do what they like in their Ministries."6. Er stellte das alleinige politische Entscheidungszentrum dar und befaßte sich oft auch mit Banalitäten. Seine Kabinettsminister, die er öffentlich als "my boys" abzukanzeln pflegte, und leitende Beamte unterwarf er einer permanenten Ämterrotation (um ihnen den Aufbau einer eigenen Machtbasis unmöglich zu machen). 7

Zu mächtig gewordene Politiker wurden geschaßt, eingekerkert oder umgebracht. Kritik an Entscheidungen Bandas zu üben, war unmöglich. Er verfügte durch seine mächtige Einheitspartei "Malawi Congress Party" (MCP), die - in afrikanischen Staaten nicht selbstverständlich - bis hinunter auf die Dorfebene organisiert ist, über ein effizientes Kontroll- und Disziplinierungsinstrument.

Alle wichtigeren und wichtigen Emennungen in öffentliche Ämter in Regierung und Verwaltung nahm Banda selbst vor. In den Parlaments"wahlen" von 1964, 1971 und 1976 wurde für jeden Wahlkreis nur ein einziger Kandidat aufgestellt, den Banda in seiner

4 Eine Diskussion über die Demokratisierung afrikanischer Staaten würde den Rahmen dieses Beitrages sprengen. Einen guten Überblick über den Forschungsstand zu diesem Thema bietet Siegmar Schmidt, 1993: Demokratisienungsprozesse in Afrika, in: Merkel, Wolfgang (Hrsg.): Systemwechsel 1, Opladen.

5 Vgl. Weltbank, 1993: Weltentwicklungsbericht 1993, Washington D.C., Tab. 1, S. 290.

6 Zitien bei Heiko Meinharde, 1993: Die Rolle des Parlaments im autoritären Malawi, Hamburg, S. 36.

7 Vgl. Meinhardt, 1993, a.a.O., S. 100-105. 
Eigenschaft als Präsident auf Lebenszeit der MCP vorher ausgewählt hatte. Mangels Konkurrenz wurde der eigentliche Wahlgang entbehrlich. Erst in den Parlamentswahlen von 1978, 1983, 1987 und zuletzt 1992 hatten die Wähler in vielen Wahlkreisen die Auswahl zwischen zwei und fünf Kandidaten, wobei auch hier alle potentiellen Abgeordneten vorher von Banda persönlich zugelassen sein muBten. Das Interesse an diesen Wahlen blieb gering, wobei keine Angaben über die Beteiligung erhältlich sind. 8

Banda selbst brauchte sich bisher keiner Volkswahl zu stellen. Er lieB sich vom Parteikonvent der MCP 1970 zum Staatspräsidenten auf Lebenszeit wählen und dieses Privileg in der Verfassung festschreiben.9

Oppositionelle hatten keine Chance, sich innerhalb Malawis zu organisieren. Lediglich im benachbarten Ausland hielten sich verschiedene kleine, überwiegend sozialistisch orientierte und in ihrer Anhängerschaft als eher unbedeutend einzustufende Oppositionsparteien auf, wie z.B. die "LESOMA" (Socialist League of Malawi) und die "MAFREMO" (Malawi Freedom Movement), die vom im Oktober 1992 in einem malawischen Gefängnis verstorbenen ehemaligen Justizminister Orton Chirwa in Dar es Salaam gegründet wurde.10

Zu einer ersten ernsten Herausforderung des Banda-Regimes kam es erst im März 1992, als die katholischen Bischöfe des Landes in einem Hirtenbrief harsche Kritik an der Diktatur übten und u.a. eine Wahrung der Menschen- und Freiheitsrechte einforderten.

Die öffentliche Kritik des Klerus an dem Banda-Regime traf nicht nur die Regierung überraschend (unmittelbar nach der Aktion wurde auf einer eilig einberufenen Sitzung des Exekutivkommitees der MCP, dem auch viele Kabinettsminister angehören, die Ermordung der Bischöfe beschlossen. Banda - unter internationalem Druck - verwarf jedoch diesen Plan.11), sondern auch die Öfentlichkeit. Obwohl der Besitz und die Weitergabe des Hirtenbriefes unter Strafe gestellt wurde, lieB sich die ins Rollen gebrachte Lawine nicht mehr halten. Im Nachbarland Zambia schlossen sich im März 1992 die wichtigsten Oppositionsgruppen im Exil unter der Führung des Generalsekretär des "Southem African Trade Union Cooperation Council" (SATUCC), Chakufwa Chihana zur "United Front for Multiparty Democracy (UFMD) zusammen. Einen Monat später forderte Chihana mit seiner medienwirksamen Rückkehr nach Malawi das Regime offen heraus. Seine sofortige Verhaftung und ein besonders von den Geberstaaten beobachteter Prozeß mit anschließender Verurteilung wegen Auf wiegelung stilisierten den Gewerkschafter nicht nur im Ausland zu

8 Vgl. Meinhardt, 1993, S. 75-84.

9 Constitution of Malawi, Chapter III, Art.9.

10 Vgl. Meinhardt, 1993, S. 61-67. Zur Situation der Menschenrechte siehe: Africa Watch, 1990: Where Silence Rules. The Suppression of Dissent in Malawi, New York et.al.

11 Von der Sitzung gibt es einen geheimen Tonbandmittschnitt, der dem Autor zugänglich war. Banda selbst nahm an der Sitzung nicht teil. 
einer Art Märtyrer, obwohl sein Bekanntheitsgrad in Malawi zu diesem Zeitpunkt nicht sehr groß war.12

Anfang Mai 1992 kam es erstmals seit der Unabhängigkeit zu Streiks und Massenprotesten in den Städten und auf einigen Plantagen, die nicht in etster Linie politisch, sondem ökonomisch bedingt waren. Sie wurden vom Sicherheitsapparat blutig beendet.

Úber diese endogenen Faktoren hinaus, die das Banda-Regime unter Druck setzten, hatten besonders die westlichen Geberstaaten und ihre Institutionen (Weltbank, IWF) entscheidenden Einfluß auf die politische Entwicklung. Im Mai 1992 wurde von den Geberstaaten eine Suspendierung der Finanzhilfen beschlossen und ihre Wiederaufnahme von Fortschritten bei der Demokratisienung des autoritären Regimes abhängig gemacht.13 Diese politische Konditionierung der Finanzhilfe traf das Regime besonders schwer, da die öffentliche Entwicklungshilfe stolze 22,6 \% des Bruttosozialproduktes (1991) ausmachte. 14 Der jahrelang hofierte Günstling des Westens, der als Garant des Antikommunismus galt und sowohl enge Beziehungen zur früheren Kolonialmacht Großbritannien als auch - als einziger afrikanischer Staat - diplomatische Beziehungen zum südafrikanischen Apartheidsregime pflegte, wurde somit ein Opfer der veränderten Weltlage nach dem Zusammenbruch des Kommunismus. Die Geberstaaten waren nicht länger bereit, Menschenrechtsverletzungen und Autoritarismus billigend in Kauf zu nehmen.

Im Juni 1992 wurden fällige Parlamentswahlen im alten Stil durchgeführ. In 91 der 141 Wahlkreise $(64,5 \%)$ gab es eine Kandidatenkonkurrenz (freilich nur innerhalb der Einheitspartei MCP), während in den restlichen Wahlkreisen nur ein (31,9\%) oder gar kein Kandidat (3,6 \%) zur Wahl stand. Bei einer Wahlbeteiligung von vermutlich nicht mehr als 20-30\% der registrierten Wähler kam es zu einer relativ hohen Austauschrate von $65 \%$, die - neben der geringen Wahlbeteiligung - ein weiteres Zeichen für die Unzufriedenheit der Bevölkerung mit der Regierung ist. Da abgewählte Kabinettsmitglieder anschließend von Banda wieder in ihre Ämter ernannt wurden, wurde die Wahl gänzlich zur Farce. 15

Das Regime versuchte, durch kleine, vorsichtige, oft nur kosmetische Reformen die Geberstaaten zu beruhigen und den innenpolitischen Druck einzudämmen. So wurde Anfang Oktober 1992 ein Präsidialkomitee ("Presidential Committee on Dialogue" (PCD)), dem einige Mitglieder des Exekutivkomitees der MCP und ausgewählte weitere Kabinettsmitglieder angehören, gegründet, das mit oppositionellen Kräften, wie Kirchen, Freiberuflern

12 Zur Chronik der Ereignisse vgl. Goswin Bawnhögger, 1993: Malawi, in: Hofmeier, Rolf / Institut für Afrika-Kunde (Hrsg.): Afrika Jahrbuch 1992, Hamburg, S. 328-337.

13 Frankfurter Rundschau, 14.05.1992.

14 Vgl. Weltbank, 1993: Weltentwicklungsbericht 1993, Washington D.C., Tabelle 20, S. 328.

15 Vgl. Meinhardt, 1993, S. 75-84. 
und Mitgliedem der im Untergnund arbeitenden Gruppierungen "Aford" (Chihanas Organisation Alliance for Democracy) und United Democratic Front (UDF) unter dem geschaßten ehemaligen MCP-Funktionär Bakili Muluzi, die sich zum "Public Affairs Committee" (PAC) zusammengeschlossen hatten, über demokratische Reformen verhandeln soll.

Um den internen und externen Druck zu entschärfen, wurde in der Umgebung von Banda die Abhaltung eines Referendums über das Parteiensystem vorgeschlagen. Obwohl sein Ausgang in den Machtzirkeln der MCP als ungewiB eingeschätzt wurde, haben anscheinend führende Politiker Banda, der als über 90 jähriger Greis von der Außenwelt ziemlich abgeschirmt war, über seine Popularität im Lande bewußt im unklaren gelassen. 16 Banda - in der (verhängnisvollen) Gewißheit, das Referendum ohne Probleme gewinnen zu können stimmte dem Volksvotum im Oktober 1992 zu. Schon wenig später wurde die Verfassung entsprechend geändert.

Der zunächst geplante Termin im März 1993, mit dem er offensichtlich beabsichtigt hatte, die Artikulations- und Organisationschancen der Oppositionsgruppen stark einzuschränken und - wie z.B. Felix Houphouet-Boigny in der Côte d'Ivory bei den frühen Parlaments- und Präsidentschaftswahlen (November 1990) - den Ausgang zu seinen Gunsten auszunutzen, wurde auf internen und internationalen Druck auf den 14. Juni 1993 verschoben.

\section{Das Referendum vom 14. Juni 1993}

\subsection{Im Vorfeld des Referendums: Organisation und Artikulation gesellschaftlicher Interessen}

Die Position der MCP Bandas war klar. Sie kämpfte geschlossen für eine Mehrheit für die Beibehaltung des Einparteiensystems im Referendum. Die gewaltige Mobilisierungsmaschinerie der mächtigen Partei, die über einen erstaunlich hohen Organisationsgrad verfügt und bis hinunter auf die Dorfebene landesweit effektiv - und nicht nur auf dem Papier - vertreten ist, wurde aktiviert. Úberall im Lande wurde aggressiv für das Einparteiensystem, das sich seit fast 30 Jahren bewährt habe, geworben. Alle hohen Regierungs- und Parteifunktionäre wurden bis in die letzten Winkel Malawis entsandt, um die Wähler für ihre Sache zu gewinnen.

16 Interviews mit dem Mitglied des Exekutivrates der MCP, A, am 23.06.1993 und dem ehemaligen Kabinettsmitglied B am 21.06.1993. Beide waren bei den Konsultationen mit Banda anwesend. Aus prinzipiellen Gründen werden die Gesprächspartner in diesem Aufsatz nicht preisgegeben, sondem durch Großbuchstaben unterscheidbar gemacht. 
Taktisch ungeschickt handelte Präsident Banda, als er sich als Zugpferd der MCP öffentlich auf ein Einparteiensystem festlegte. Als Stastschef hätte er sich mehr Handlungsfreiheit bewahren können, wenn er sich aus dem Wahlkampf herausgehalten hätte. Die enge Verknüpfung seiner eigenen Person mit dem Einparteiensystem mußte bei einer Niederlage auch indirekt als Niederlage Bandas gelten. 17

Schon während des Wahlkampfes mußten die regionalen und lokalen MCP-Funktionäre alle Register ziehen, um Anhänger zu mobilisieren. Das geschah in Gegenden der Zentralregion einerseits durch Korrumpierung der traditionellen Dorfvorsteher und Chiefs, die gegen Geldbeträge in bar ihre Dörfer für ein Votum für die Beibehaltung des Einparteiensystems mobilisieren sollten. Die Folge war, daß manche Dörfer geschlossen in das Wahlregister aufgenommen wurden. Andererseits gab es dort auch massive Einschüchterungen, etwa in Form der sog. Nyau-Tänzer, die traditionell einer Art schwarzen Magie verbunden, heute jedoch im Auftrag der MCP in furchterregende Masken und traditionelle Gewänder gehüllt, Aktivisten der Oppositionsgruppienmgen ver jagen und ihre Anhänger einschüchtem wollten.18 In den Distrikten mit den riesigen Tabakplantagen (Malawis Hauptexportgut), die fast ausschließlich dem Präsidenten und seinen engsten Günstlingen gehören, waren Einschüchterungen besonders häufig an der Tagesordnung. Mehrparteienanhängem wurde eine Lohnkürzung oder sogar der Verlust des Arbeitsplatzes angedroht. 19

Die MCP war auch noch in anderen Bereichen privilegiert. So wurde es Staatsbeamten gestattet, während der bezahlten Dienstzeit an MCP-Veranstaltungen teilzunehmen, während jene, die Kundgebungen der Opposition besuchen oder während der Wählerregistrienung als Monitore der oppositionellen Gruppen wirken wollten, eine fünfzigprozentige Lohnkürzung für ihre Ausfallzeiten akzeptieren mußten.20 Auch der Zugang zu den Medien war unausgewogen. Es gibt zwar seit Anfang 1993 eine ständig wachsende Anzahl von regimekritischen Zeitungen, die aber alle aus Geldmangel und vor allem aus Mangel an Druckereien nur unregelmäßig oder höchstens zweimal wöchentlich erscheinen können. Die einzige Tageszeitung des Landes, die Daily Times, gehört zum Finanzimperium Bandas. Auch der Zugang zur einzigen Rundfunkstation "Malawi Broadcasting Corporation" (MBC), die in staatlicher Hand ist, war für die Oppositionsgruppen stark eingeschränkt. Während über Veranstaltungen der MCP oft detailliert berichtet wurde, mußte

17 Seine auf vielen Kundgebungen wiederholte Frage "Do you still want your Kamuzu (Präsident Banda)?" macht die enge Verquickung seiner Person mit dern Einparteiensystern besonders deutlich.

18 Vgl. auch Thania Pfaffenholz, 1993: Votum für Pluralismus, in: Informationsdienst südliches Af rika, 4/93, S. 16-18.

19 Der Autor war als offizieller Wahlbeobachter in Mchinji und erfuhr den Sachverhalt in zahlreichen Gesprächen.

20 Confidential Report of the Joint Intemational Observer Group (JOG) of the United Nations, Southem Region, Lilongwe. 
sich die Opposition mit der Bekanntgabe von On und Zeit ihrer Kundgebungen zufriedengeben.21 Darüber hinaus hatte Banda als Staatschef als einziger Politiker direkten Zugang zur MBC. Seine Reden wurden stets ausgestrahlt.

Gegen Ende der Registrierungsphase kam es zu zahlreichen Umbesetzungen der Distriktverwaltungschef s, womit vermutlich sichergestellt werden sollte, daß dem Regime loyale Kräfte in diesen wichtigen Positionen zur Verfügung standen.22

Ein gravierender Nachteil für die Oppositionsgruppen bestand darin, daß die Genehmigung für jede Art von Kundgebung von den örtlichen Polizeibehörden eingeholt werden mußte. Das bedeutete de facto, daß alle MCP-Veranstaltungen prompt genehmigt wurden, während mit den Anmeldungen der Opposition oft willkürlich verfahren wurde. Entweder wurde die Erlaubnis erst sehr spät oder - aus angeblichen Sicherheitserwägungen - gar nicht erteilt. Das galt besonders für zahlreiche ländliche Gegenden. Wenn Präsident Banda, der in einer einzigartigen Wahltoumee alle 25 Distrikte des Landes besuchte, sein Kommen ankündigte, wurden kurzerhand schon genehmigte Oppositionsveranstaltungen im entsprechenden Distrikt verboten.23 Aus Mangel an Ressourcen komnten die Oppositionsgruppierungen auch nicht in dem Maße wie die Regierungspartei Wahlkampf betreiben. Es steht zu vermuten, daß die MCP auch staatliche Ressourcen eingesetzt hat 24 und Spenden in beträchtlicher Höhe von mindestens einem multinationalen Konzern erhalten haben soll.25

Oppositionsveranstaltungen wurden nicht selten auch durch die "Malawi Young Pioneers", einer paramilitärischen Organisation, gestört.

Ob die späte Freilassung des Oppositionspolitikers und Führers der "Aford", Chihana, unmittelbar vor dem Referendum die Chancen der Anhänger des Mehrparteiensystems geschmälert hat (wie vermutlich von der MCP erhofft), muß zurückhaltend eruiert werden. Zwar fiel er als aktiver Wahlkämpfer aus, aber seine Inhaftierung hatte eine sicherlich wichtige Symbolfunktion, die ihm zusätzliche Unterstützung eingebracht haben dürfte.

21 Confidential Report der JOG, Lilongwe.

22 Intemes Papier der JIOG, Mai 1993.

23 Report of JIOG on the Malawi National Referendum, August 1993, Lilongwe.

24 Einer vertraulichen Information zufolge soll Banda beträchtliche Kredite von Geschäftsbanken aufgenornmen haben, die zu seinem eigenen Finanzimperium Press Holdings gehören.

25 Tiny Rowland, der Chef des Lonho-Konzems und guter Freund Bandas, soll der MCP für ihre Referendumskampagne ca. 675.000 US\$ gespendet haben. Vgl.: Southem Af rica Political \& Economic Monthly, 1993, 7, 2, S. 24. 
Wichtig ist die Tatsache, daß es offenbar im Vorfeld des Referendums nicht nur zu politisch motivierten Einschüchterungen, sondem auch zu Morden durch Einheiten der "Jungen Pioniere" gekommen ist.26

Inhaltlich zeichneten sich die Veranstaltungen durch gegenseitige Diffamierungen aus. Die Opposition bediente sich häufig älteren Zitaten Präsident Bandas, um ihn lächerlich zu machen. Alte Mißstände, besonders der sehr unbeliebte (und Mitte 1992 eingestellte) Zwang, einen Mitgliedsausweis der MCP besitzen, oder Zwangsspenden für die Privatschatulle Bandas leisten zu müssen, wurden aufgegriffen. Die Regierung wurde für die ökonomische Misere verantwortlich gemacht, und mit kaum realisierbaren Versprechungen für große Teile der Bevölkerung wurde nicht gespart.

Die MCP hatte kaum Anstrengungen zur politischen Emeuerung gezeigt. Der Wahlkampf war ganz auf die Persönlichkeit Bandas zugeschnitten und paralysierte somit inhaltliche Diskussionen weitgehend. Wie ublich wurden die Reden Bandas rezipiert und seine Verdienste um die Unabhängigkeit und sein Beitrag zur Entwicklung Malawis gelobt. Die Zwangsspenden und Parteikartenpflicht wurden als Fehler eingeräumt, die jedoch ohne Wissen des Präsidenten von übereifrigen MCP-Funktionären begangen worden seien 27 (was kaum überzeugend ist). Ansonsten ähnelten die Veranstaltungen nicht selten einem Volksfest: Frauen in der Nationaltracht mit dem Konterfei Bandas (als Jugendportrait) führten traditionelle Tänze vor und sangen Loblieder auf den Präsidenten.

Uberschattet wurde die Vorbereitungsphase zum Referendum durch einen scharfen Disput zwischen Regierung und Opposition in der Frage der Anzahl der Wahlumen. Das Regime beabsichtigte, je eine Ume für Voten zum Erhalt bzw. gegen die Fortsetzung des Einparteiensystems zu installieren, während die Opposition auf eine Wahlume bestand. Da die vom Regime präferierte Möglichkeit die Manipulationschancen erhöht hätte (da die Umen offen sichtbar aufgestellt werden sollten, wäre die Stimmenabgabe nicht mehr geheim gewesen; außerdem lassen sich getrennte Wahlumen leichter austauschen), drohten die Oppositionsgruppen mit einem Boykott des Referendums, was einem Scheitem gleichgekommen wäre und weitere Sanktionen der Geberländer bedeutet hätte. Unter intemationaler Vermittlung akzeptierte Banda erst sehr spät (ca. einen Monat vor dem Wahltag) das Aufstellen nur einer Wahlume.28

Um einer Politisierung der Sicherheitskräfte vorzubeugen, wurden das Militär und die Polizei - wie schon bei den Wahlen zum Einparteienparlament - von der Abstimmung ausge-

26 Der Autoruntersuchte zusammen mit einem Kollegen einen solchen Fall vor Or.

27 Das wurde auch in einem Interview mit dem Minister E (01.07.1993) deutlich.

28 The Times, 19.05.1993. 
schlossen. ${ }^{29}$ Die "Jungen Pioniere", eine paramilitärische Organisation, die Repressionsaufgaben für das Regime wahrgenommen hat, war allerdings von dem Verbot ausgenommen.

Ansonsten durften sich alle malawischen Staatsbürger, die mindestens 21 Jahre alt sind, an dem Referendum beteiligen. 30

Die Registrierung der Wähler verlief unter internationaler Aufsicht und wurde im großen und ganzen als frei und fair bezeichnet. Technische Probleme gab es mit der Behandlung von Registrienmgsdokumenten von den Parlamentswahlen 1992. Nicht allen Registrierungsbeamten war bekannt, daß diese auch für das Referendum als gültig anerkannt werden sollten. So kam es in einigen Gegenden zu Doppelregistrierungen. 31

\subsection{Der Wahlvorgang: Möglichkeiten und Grenzen eines Volksvotums}

In der ersten freien Wahl seit der Unabhängigkeit 1964 hatten 4,7 Millionen registrierte Wähler die Möglichkeit, in uber 2000 Wahlzentren für die Beibehaltung des Einparteienoder die Einfühnung eines Mehrparteiensystems zu votieren.

Jedes Wahlzentrum bestand aus einem bis zu uber zehn Wahllokalen, in denen jeweils bis zu tausend Wähler registriert waren. Durch diese Aufteilung sollte sichergestellt werden, daß kein Wähler mehr als fünf Kilometer zuracklegen mußte, um sein Wahllokal zu erreichen. 32

Als Wahlzentren dienten fast ausschließlich Schulgebäude. In den Klassenzimmern wurden in Malawi gefertigte Wahlkabinen aufgestellt, um eine geheime Abstimmung zu garantieren.

Technisch war der Wahlvorgang wie folgt organisiert: Der Wähler mußte sein Registrierungsdokument (aus dem auch das für ihn zuständige Wahllokal ermittelt werden konnte) den Wahlhelfern vorlegen. Wenn sein Name auch im Wahlregister vermerkt war, wurde er zur Abstimmung zugelassen. Um Manipulationen ("Mehrfachwählen") zu verhindem, wurde ein Finger der rechten Hand mit nichtabwaschbarer Tinte markiert. Dann bekam der

29 In den Streitkräften wurde diese Maßnahme anscheinend begrüßt. Politische Konflikte innerhalb des Militärs sollten auf diesem Wege verhindert werden. Interview mit dem Offizier C, 02.07.1993.

30 Constitution (Referendum on Malawi's Political System) Regulations, 1993, Part III, 14.

31 Intemer Bericht der JOG.

32 Report of the Joint Intemational Observer Group on the Malawi National Referendum, I ilongwe, August 1993. 
Wähler zwei Stimmzettel (einer zeigte einen schwarzen Hahn, das Symbol der MCP und Symbol für die Präferenz des Einparteiensystems, der andere eine Lampe als Symbol für das Mehrparteiensystem) und einen Briefumschlag. In der Wahlkabine befand sich ein aus Pappe gefertigter verschlossener Behälter, in den der nicht benutzte Stimmzettel gesteckt werden sollte. Der präferierte Stimmzettel wurde in den Umschlag gesteckt, verschlossen und dann unter den Augen des Wahlpersonals in die Wahlurne gesteckt. Durch eine zweite Tür (falls vorhanden) verließ der Wähler das Wahllokal.

In jedem Wahllokal waren mehrere Wahlhelfer - meistens Lehrer - und nationale Beobachter anwesend. Häufig war die MCP mit zwei Repräsentanten vertreten, während die UDF, Aford und PAC oftmals zusammen nur zwei Beobachter stellten (was an der noch rudimentären Organisationsstruktur, Finanzmangel und dünnen Personalschicht der Oppositionsgruppen gelegen hat). ${ }^{33}$ Als Wahlleiter fungierte in jedem Wahlzentrum ein Staatsbeamter (meistens Schulleiter oder Sekundarschullehrer). Ein nicht bewaffneter Polizist sorgte für Ordnung.

210 intemationale Beobachter, die die "Joint Intemational Observer Group" (JIOG) bildeten, waren in jedem der 25 Distrikte im Einsatz und besuchten zahlreiche Wahlzentren.

Die Wahllokale waren von 6.00 bis 18.00 Uhr durchgehend geöffnet. Nach Schließung wurden zunächst die Pappbehälter mit den weggeworfenen Stimmzetteln öffentlich verbrannt. Danach wurden die verschlossenen Wahlurnen - unter Anwesenheit der nationalen und (falls anwesend) der internationalen Beobachter - nacheinander geöffnet und ausgezählt.34 Die Stimmzettel wurden soriert (schwarzer Hahn, Lampe und ungültig) in Plastiktüten verpackt und versiegelt, das Auszählungsergebnis in Formulare eingetragen. Spät in der Nacht oder auch erst am (frühen) Morgen wurden die Stimmzettel und Ergebnisbogen zur zuständigen Distriktverwaltung gebracht. Dort wurden die Wahlergebnisse unter Aufsicht internationaler Beobachter gesammelt und ausgewertet. Die Ergebnisse wurden der Wahlkommission mitgeteilt.

Das Referendum lief überraschend reibungslos ab. Schon bei Öfnung konnte vor vielen Wahllokalen ein reger Andrang festgestellt werden. Einige Wähler warteten seit $3.00 \mathrm{Uhr}$ nachts diszipliniert eingereiht auf die Öfnung. Ab dem frühen Nachmittag verebbte der Andrang, so daß ab ca. 16.00 Uhr das Wahlpersonal oft unter sich blieb. Die Wähler wollten vermutlich rechtzeitig vor Einbruch der Dunkelheit wieder in ihren Dörfern sein.

33 Den nationalen Beobachtem wurde von ihrer Organisation ein Salär gezahlt. Die UDF zahlte 100 Kwacha (K), ca. 40.- DM, die MCP K 75. Es soll auch vorgekornmen sein, daß ein Monitor sich von beiden Seiten verpflichten ließ, was die Funktion der nationalen Beobachtung in solchen Fällen faktisch aufhob. Interview mit B, 03.07.1993.

34 Da die meisten Wahlzentren nicht an das Stromnetz angeschlossen sind, fand die Auszählung unter Taschenlampenlicht (Von der Wahlkommission zur Verfügung gestellt) statt. 
Obwohl es kleinere Zwischenfälle gab (im Mulanje District mußten z.B. mindestens 3000 Wähler weggeschickt werden, da die Stimmzettel ausgegangen waren), hatten diese nach Sicht der JOG keine signifikanten Auswirkungen auf das Ergebnis. 35 In Dowa traten die "Jungen Pioniere" geschlossen zur Abstimmung an und waren nicht gewillt, sich in die Wählerschlangen einzureihen. Die Anwesenheit von intemationalen Beobachtem stimmte sie schließlich um.

Einschüchterungsversuche gab es besonders in der Zentralregion. Dort wurden Wähler gezwungen, an der Wahl teilzunehmen und für das Einparteiensystem zu stimmen. Als Beweis für ihre "Loyalität" verlangten einige Dorfhäuptlinge anscheinend die Vorlage der "Lampe". Obwohl Stimmzettel nicht aus dem Wahllokal mitgenommen werden durften, konnte das nicht kontrolliert werden.36 Bei Nichtbefolgen wurden Sanktionen angedroht (Verlust von Ackerland, Arbeitsplatzverlust auf den Plantagen).

Probleme gab es häufig mit älteren Leuten, denen der Wahlvorgang teilweise völlig unklar war. Hier konnte das Wahlpersonal aber meistens Abhilfe schaffen.

Die nationalen Beobachter - auch die der Oppositionsgruppen - verhielten sich häufig sehr passiv und äußerten den intemationalen Kollegen gegenüber kaum irgendwelche Kritik oder Anregungen. Das ist vermutlich darauf zurückzuführen, daß sie ihre Aufgabe in erster Linie als "Job" sahen. Andererseits wirkte vielleicht die Anwesenheit der MCP-Funktionäre einschüchtemd.

\subsection{Detailanalyse des Ergebnisses}

Wie aus Tabelle 1 ersichtlich ist, haben sich respektable Zweidrittel der registrierten Wähler an dem Referendum beteiligt und mit $63 \%$ gegen $35 \%$ für die Einführung eines Mehrparteiensystems votiert, obwohl - wie oben dargestellt - die Oppositionsgruppen gegenüber dem Regime in vielerlei Hinsicht benachteiligt waren.

Das Ergebnis weist einige regionale Besonderheiten auf, die von analytischer Bedeutung sind: So ist ein regional unterschiedliches Wahlverhalten festzustellen. Während in der Nord- und Südregion hohe Mehrheiten für das Mehrparteiensystem erzielt wurden, konnte sich das Einparteiensystem mit einer knappen Zweidrittel-Zustimmung in der Zentralregion durchsetzen. Hier konnte die Opposition nur in einem Distrikt (Ntcheu) eine deutliche Mehrheit erzielen. Das ist darauf zurückzuführen, daß dort die zahlenmäßig kleine Ethnie

35 Report of the JIOG on the Malawi National Referendurn, Lilongwe, August 1993.

36 Besonders alte Frauen waren überrascht, als sie vorn Wahlhelfer darüber aufgeklärt wurden, da $B$ sie auch für die "Lampe" (also für das Mehrparteiensystem) votieren konnten. 
Tabelle 1: Registrierte Wähler

\begin{tabular}{|c|c|c|c|c|c|}
\hline Distrikt & 1987 & 1992 & 1993 & $\begin{array}{l}87 / 93 \\
(\%)\end{array}$ & $\begin{array}{c}92 / 93 \\
(\%)\end{array}$ \\
\hline North & 381.287 & 449.900 & 629.339 & 65.1 & 39.9 \\
\hline $\begin{array}{l}\text { Chitipa } \\
\text { Karonga } \\
\text { Nkhata Bay } \\
\text { Rumphi } \\
\text { Mzuzu (*) } \\
\text { Mzimba }\end{array}$ & $\begin{array}{r}41.057 \\
80.771 \\
81.816 \\
39.827 \\
137.816\end{array}$ & $\begin{array}{r}52.200 \\
70.300 \\
65.700 \\
46.500 \\
\\
\\
215.200\end{array}$ & $\begin{array}{r}58.404 \\
110.603 \\
129.514 \\
59.300 \\
110.980 \\
160.538\end{array}$ & $\begin{array}{l}42.3 \\
36.9 \\
58.3 \\
48.9 \\
97.0\end{array}$ & $\begin{array}{l}11.9 \\
57.3 \\
97.1 \\
27.5 \\
\\
26.2\end{array}$ \\
\hline Central & 1.335 .839 & 1.561 .100 & 1.833 .820 & 35.3 & 17.5 \\
\hline $\begin{array}{l}\text { Dedza } \\
\text { Dowa } \\
\text { Kasungu } \\
\text { Lilongwe } \\
\text { Mchinji } \\
\text { Nkhotakota } \\
\text { Ntcheu } \\
\text { Ntchisi } \\
\text { Salima }\end{array}$ & $\begin{array}{r}246.257 \\
146.919 \\
110.737 \\
442.472 \\
71.426 \\
55.961 \\
118.396 \\
79.251 \\
84.420\end{array}$ & $\begin{array}{r}198.000 \\
151.500 \\
182.600 \\
477.100 \\
128.400 \\
86.800 \\
181.800 \\
57.400 \\
97.500\end{array}$ & $\begin{array}{r}184.589 \\
194.010 \\
232.276 \\
591.460 \\
196.393 \\
133.866 \\
142.550 \\
64.204 \\
94.472\end{array}$ & $\begin{array}{r}-25.0 \\
32.1 \\
109.8 \\
33.7 \\
175.0 \\
139.2 \\
20.4 \\
-19.0\end{array}$ & $\begin{array}{r}-6.8 \\
28.1 \\
27.2 \\
24.0 \\
53.0 \\
54.2 \\
-21.6 \\
11.9 \\
-3.1\end{array}$ \\
\hline South & 1.541 .781 & 2.009 .600 & 2.236 .367 & 11.9 & 11.3 \\
\hline $\begin{array}{l}\text { Blantyre } \\
\text { Chikwawa } \\
\text { Chiradzulu } \\
\text { Machinga } \\
\text { Mangochi } \\
\text { Mulanje } \\
\text { Mwanza } \\
\text { Nsanje } \\
\text { Thyolo } \\
\text { Zomba }\end{array}$ & $\begin{array}{r}196.151 \\
125.237 \\
95.770 \\
194.664 \\
162.288 \\
279.153 \\
33.508 \\
86.946 \\
169.497 \\
198.567\end{array}$ & $\begin{array}{r}305.600 \\
163.100 \\
98.100 \\
265.500 \\
275.900 \\
305.100 \\
61.900 \\
113.800 \\
205.100 \\
215.500\end{array}$ & $\begin{array}{r}271.152 \\
194.987 \\
98.605 \\
344.753 \\
352.263 \\
347.006 \\
74.890 \\
106.287 \\
186.262 \\
260.163\end{array}$ & $\begin{array}{r}38.2 \\
55.7 \\
3.0 \\
77.1 \\
117.1 \\
24.3 \\
123.5 \\
22.2 \\
9.9 \\
31.0\end{array}$ & $\begin{array}{r}-11.3 \\
19.6 \\
0.05 \\
29.9 \\
27.7 \\
13.7 \\
21.0 \\
-6.6 \\
-9.2 \\
20.7\end{array}$ \\
\hline Total & 3.278 .907 & 4.020 .600 & 4.699 .526 & 43.3 & 16.9 \\
\hline
\end{tabular}

Quelle: JIOG, für die Jahre 1987 und 1992: Republic of Malawi, Electoral Commission, 1987, 1992, Zomba.

(*) Bis 1992 gehörte Mzuzu zum Distrikt Mzimba. 


\begin{tabular}{|c|c|c|c|c|c|c|}
\hline $\begin{array}{r}\text { WZ } \\
1987\end{array}$ & $\begin{array}{c}\text { WZ } \\
1993\end{array}$ & $\begin{array}{c}87 / 93 \\
(\%)\end{array}$ & $\begin{array}{l}\text { EPS } \\
(\%)\end{array}$ & $\begin{array}{l}\text { MPS } \\
(\%)\end{array}$ & $\begin{array}{c}\text { nichtig } \\
\text { (\%) }\end{array}$ & $\begin{array}{l}\text { WB } \\
(\%)\end{array}$ \\
\hline 228 & 420 & 84.2 & 10.6 & 88.4 & 1.0 & 70.6 \\
\hline $\begin{array}{l}36 \\
30 \\
32 \\
22\end{array}$ & $\begin{array}{r}52 \\
63 \\
50 \\
52 \\
61 \\
108\end{array}$ & $\begin{array}{r}44.4 \\
110.0 \\
56.3 \\
136.4 \\
\\
88.0\end{array}$ & $\begin{array}{r}8.8 \\
5.8 \\
7.3 \\
13.0 \\
19.1 \\
8.3\end{array}$ & $\begin{array}{l}90.5 \\
93.4 \\
91.3 \\
85.6 \\
79.8 \\
90.0\end{array}$ & $\begin{array}{l}0.7 \\
0.8 \\
1.4 \\
1.4 \\
1.1 \\
0.8\end{array}$ & $\begin{array}{l}70.3 \\
59.1 \\
46.5 \\
86.6 \\
82.8 \\
83.7\end{array}$ \\
\hline 398 & 840 & 111.1 & 65.5 & 31.5 & 3.0 & 69.3 \\
\hline $\begin{array}{l}53 \\
36 \\
56 \\
97 \\
34 \\
35 \\
37 \\
30 \\
20\end{array}$ & $\begin{array}{r}90 \\
94 \\
104 \\
106 \\
71 \\
77 \\
103 \\
58 \\
47\end{array}$ & $\begin{array}{r}69.8 \\
161.1 \\
85.7 \\
102.1 \\
108.8 \\
120.0 \\
178.4 \\
93.3 \\
135.0\end{array}$ & $\begin{array}{l}72.3 \\
83.0 \\
70.0 \\
69.4 \\
64.4 \\
51.1 \\
25.0 \\
76.8 \\
53.6\end{array}$ & $\begin{array}{l}12.8 \\
14.6 \\
27.3 \\
27.3 \\
29.7 \\
45.3 \\
73.9 \\
21.6 \\
44.0\end{array}$ & $\begin{array}{l}2.9 \\
2.5 \\
2.7 \\
3.3 \\
5.9 \\
3.6 \\
1.1 \\
1.7 \\
2.4\end{array}$ & $\begin{array}{l}75.5 \\
72.0 \\
77.3 \\
65.1 \\
59.3 \\
59.3 \\
70.8 \\
81.1 \\
83.2\end{array}$ \\
\hline 358 & 804 & 124.6 & 14.5 & 83.5 & 2.0 & 64.4 \\
\hline $\begin{array}{l}46 \\
45 \\
22 \\
39 \\
48 \\
41 \\
22 \\
22 \\
35 \\
38\end{array}$ & $\begin{array}{r}94 \\
67 \\
53 \\
110 \\
107 \\
91 \\
59 \\
57 \\
78 \\
88\end{array}$ & $\begin{array}{r}104.4 \\
48.9 \\
140.9 \\
182.1 \\
122.9 \\
122.0 \\
168.2 \\
159.1 \\
122.9 \\
131.6\end{array}$ & $\begin{array}{r}13.2 \\
23.1 \\
10.3 \\
8.6 \\
8.9 \\
19.7 \\
28.0 \\
17.2 \\
18.4 \\
13.2\end{array}$ & $\begin{array}{l}85.9 \\
75.2 \\
85.5 \\
90.0 \\
89.3 \\
75.8 \\
70.2 \\
81.4 \\
80.0 \\
85.7\end{array}$ & $\begin{array}{l}0.9 \\
1.8 \\
4.2 \\
1.4 \\
1.8 \\
4.4 \\
1.8 \\
1.4 \\
1.6 \\
0.9\end{array}$ & $\begin{array}{l}85.0 \\
54.8 \\
83.7 \\
58.4 \\
57.2 \\
55.2 \\
55.5 \\
55.4 \\
82.4 \\
65.6\end{array}$ \\
\hline 984 & 2064 & 109.8 & 34.4 & 63.2 & 2.3 & 67.1 \\
\hline
\end{tabular}

87/93 (\%), 92/93 (\%): Prozentualer Anstieg der Anzahl der registrienten Wähler zwischen 1987 und 1993 bzw. 1992 und 1993

EPS: Einparteiensystem WB: Wahlbeteiligung

MPS: Mehrparteiensystem

WZ: Wahlzentren 1987 und 1993 und Steigerung (in \%) 
der Ngoni beheimatet ist. Die regionalen Unterschiede im Wahlverhalten deuten generell auf eine ethnische Orientierung des Abstimmungsverhaltens hin. Die Tumbuka im Norden und die Yao, die im Süden dominant sind, haben für das Mehrparteiensystem gestimmt, während die Chewa in der Zentralregion, zu denen Präsident Banda gehört, die MCP unterstützten. Seine dortige Klientel hatte Banda in entwicklumgspolitischen Entscheidungen häufig begünstigt. So wurde 1975 die Hauptstadt von Zomba (Süden) nach Lilongwe (Zentral) verlegt und ebenso der intemationale Flughafen.

Die ethnische Komponente des Wahlverhaltens läßt sich noch klarer abgrenzen. Die nach dem Referendum legalisierten politischen Parteien orientieren sich an ethnischen Linien. Die Aford hat im Norden ihre größte Anhängerschaft, während die UDF im Süden und die MCP in der Zentralregion die größte Unterstützung findet (vgl. Kapitel 4).

In der Nord- und Südregion scheinen jeweils kaum mehr als die MCP-Funktionäre für das Einparteiensystem votiert zu haben. Interessant ist auch, daB es (mit zwei Ausnahmen) keine knappen oder knapperen Ergebnisse gibt. Das Abstimmungsverhältnis ist immer eindeutig.

Da gesonderte Ergebnisse für die vier Städte (Blantyre, Lilongwe, Zomba, Mzuzu) nicht vorliegen (die Distriktangaben schließen einen großen Teil Landbevölkerung mit ein), läßt sich nicht klar evaluieren, wie die urbanen Schichten abgestimmt haben. Es kann jedoch davon ausgegangen werden, daß von der dünnen Mittelschicht (Unternehmer, Professionals, Staatsbeamte etc.) das Mehrparteiensystem präferiert wurde.37

Die Anzahl der ungültigen Stimmen liegt im Durchschnitt recht niedrig, wenn in Betracht gezogen wird, daß die Analphabetenrate bei 58,7\% (1987)38 liegt. Der geringe Anteil ungültiger Stimmen im Norden ist auf die traditionell bessere Ausbildung der Tumbuka durch Missionsschulen zurückzuführen. Die auffällig hohen Anteile ungültiger Stimmen in Mchinji, Mulanje und Chiradzulu sind jedoch nicht mit der Alphabetisierung zu erklären. Eine positive Korrelation ist nicht auszumachen, da diese Distrikte in Bezug auf Alphabetisierung eher im Mittelfeld liegen. Es gibt jedoch zwei andere Erklärungsvarianten: Entweder handelt es sich um bewußt ungültig gemachte Stimmen (der Wähler, der nicht die MCP unterstützen, sich aber auch nicht möglichen Repressalien seines Dorfhäuptlings aussetzen wollte, konnte z.B. einen leeren Umschlag abgeben und die "Lampe" als Loyalitätsbeweis vorlegen) oder die Erklärung des Wahlablaufes war besonders unzureichend. Für die erste Argumentation spräche die Tatsache, daß es sich bei Mchinji und Mulan je um Distrikte mit zahlreichen Plantagen handelt. Die Arbeiter sind - wie dargestellt - massiv eingeschüchtert

37 Sogar in den Ministerien zeigte man sich dem Autor gegenüber erleichtent über das Endergebnis, was nicht zwangsläufig besagt, daß die Beamten auch für das Mehrparteiensystem votient haben.

38 Malawi Population and Housing Census, 1987, Zomba. 
worden. Dennoch sollte diese Interpretation nicht überbewertet werden, da andere Plantagen-Distrikte keine übergroße Anzahl ungültiger Stimmen aufweisen.

Interessant ist die Entwicklung der Anzahl registrierter Wähler, ausgehend von den Parlamentswahlen 1987 bzw. 1992. Im Zeitraum von 1987 bis 1993 erhöhte sich ihre Zahl um über $43 \%$, was weit über dem demographischen Bevölkerungszuwachs liegt. Alleine von 1992 auf 1993 war ein Anstieg von 16,9\% zu verzeichnen. Der Vergleich zwischen der letzten Parlamentswahl im Einparteiensystem (1992) und dem Referendum ein Jahr später bietet Raum für eine Interpretation. Die uberdurchschnittliche Erhöhung der Anzahl registrierter Wähler in der Nordregion (39,9\%) läßt auf ein deutlich stärkeres Interesse der Bevölkerung am Referendum deuten, was auf eine hohe Mobilisienungsfähigkeit der Aford schließen läßt.39 In der Zentral- und Sütregion fallen die Steigerungsraten erheblich moderater aus. In jeweils drei Distrikten ist sogar ein Rückgang zu beobachten. Das kann auf Wanderungsbewegungen zwischen den Distrikten hindeuten oder auch nur darauf zurückzuführen sein, daß die Schätzungen von 1992 zu hochgegriffen waren. Auch hier wäre eine Úberinterpretation unangebracht und wenig aufschlußreich. Insgesamt läßt sich jedoch feststellen, daß das Interesse am Referendum sehr viel lebhafter als an den Parlamentswahlen war. Die Mobilisienungsfähigkeit der Oppositionsgruppen einerseits und der MCP (wenn vielleicht auch nur durch Repressionen) andererseits charakterisiert das Referendum.

Eine deutlich bessere Wahlinfrastruktur hat das Referendum aufzuweisen. Die Anzahl der Wahlzentren wurde mehr als verdoppelt. Eine Aussage darüber, ob das Votum unter faireren Bedingungen noch deutlicher für das Mehrparteiensystem ausgefallen wäre, bleibt im Bereich der Spekulation.

Erstmals in der Geschichte Malawis wurde eine Hochrechnung des Wahlergebnisses erstellt. Die Demoskopen der JOOG bedienten sich zweierlei Informationen. Erstens wurde in einigen repräsentativen Wahllokalen ( $1,84 \%$ der Wahlbevölkerung) ein "quick count" vorgenommen, der sofort an das Rechenzentrum der JIOG telefonisch übermittelt wurde 40 . Außerdem wurden bestimmte Teilergebnisse von JOG-Mitarbeitem aus den Distriktverwaltungen an das Rechenzentrum durchgegeben. Schon um 1.00 Uhr morgens (15.06.93) lag die Hochrechnung vor, die nur um 0,2\% vom amtlichen Endergebnis abwich. 41

39 Da es für die Parlamentswahlen keine veräßlichen Angaben über die Wahlbeteiligung gibt, müssen die Registrierungszahlen als Ersatz dienen.

40 Da es in den Wahlzentren fast nie funktionierende Telefone gab, konnten die Ergebnisse oft crst aus der Distriktverwaltung an die JOG-Zentrale weitergegeben werden, was $7 \mathrm{u}$ Verzögerungen führte.

41 Report of the JIOG on the Malawi National Referendum, Lilongwe, August 1993. 
Insgesamt wurde - trotz der dargestellten kleineren Vorkommnisse - das Referendum von der JIOG als frei und fair bezeichnet. 42

\section{Die politischen Folgen des Referendums}

Am 16. Juni 1993 wurde das amtliche Endergebnis der Wahlkommission bekanntgegeben, aber erst am Abend des 17. Juni nahm Präsident Banda in einer vom staatlichen Rundfunksender MBC ausgestrahlten Rede zum Referendum Stellung. Er anerkannte das Votum für ein Mehrpareiensystem und kündigte seine Legalisierung innerhalb eines Monats und allgemeine Wahlen innerhalb eines Jahres an. Trotz seiner persönlichen Niederlage (herbeigeführt durch sein starkes Engagement für die Beibehaltung des Einparteiensystems) lehnte er die Forderung der Opposition ab, zurückzutreten und einer Ubergangsregierung die Macht zu übertragen. 43 Verfassungsrechtlich stand diese Möglichkeit jedoch nicht zur Debatte, denn es wurde nicht über eine Regienung, sondem über ein politisches System abgestimmt. Die Oppositionsgruppen hatten mit ihrem Sieg im Referendum noch kein Volksvotum. Die unüberlegte Äußerung des Aford-Chefs Chihana, Banda solle ihn zum exekutiven Premierminister und Vorsitzenden einer Ubergangsregierung von Aford, UDF und MCP emennen 44 , hätte ihn fast disqualifiziert. Eine derartige Vorstellung zeigt, daß auch die Opposition nicht frei von Machthunger ist, denn die UDF ist die wahrscheinlich größere Oppositionspartei.

Das Ergebnis des Referendums ist in gewisser Weise für den Demokratisierungsprozeß günstig ausgefallen. Die MCP hat zwar eine deutliche, aber keine vernichtende Niederlage hinnehmen müssen. Sie kann sich noch immer recht gute Wahlchancen ausrechnen.

Die relativ späte Anerkennung des Ergebnisses durch Banda (nach zwei Tagen) läßt den Schluß zu, daß auch andere Szenarien durchgespielt worden sein könnten, z.B. Verhängung des Notstandes mit Verbot und Inhaftierung der Opposition. In diesem Fall hätte das Banda-Regime jegliche Entwicklungshilfe verwirkt. Aber nicht nur der intemationale Faktor scheint ausschlaggebend gewesen zu sein, sondem besonders auch die Haltung der Streitkräfte, die z.T. nach Verkündung des Ergebnisses Freudenmärsche durch einige

42 Pressemitteilung der JOG, Lilongwe, 17.06.1993. Zu einem anderen Ergebnis kamen die Wahlbeobachter der "European Parliamentarians for Sourhem Africa", die im wesentlichen die dargestellte Benachteiligung der Opposition als AnlaB nahmen, das Referendum als nicht frei und fair $\mathrm{zu}$ bewerten. Diese Argumentation erscheint mir aus praktischen Erwägungen als übertrieben, da das Ergebnis für sich spricht und nicht delegitimiert werden sollte.

43 Der Text der Rede ist abgedruckt in: Daily Times (Blantyre), 18.06.1993.

44 The Times (London), 16.06.1993. 
Städte unternahmen. Spätestens dann mußte dem Regime klar sein, daß die Verhängung des Notstandes vermutlich einen Militärputsch nach sich gezogen hätte.45

Schon am 29. Juni 1993 wurde vom Parlament, dem nur MCP-Abgeordnete angehören, das Mehrparteiensystem verfassungsrechtlich legalisiert und die von Präsident $B$ anda nach dem Referendum angekündigte Amnestie für Exilpolitiker beschlossen. 46 Beide Gesetze wurden - zusammen mit drei weiteren Vorlagen - in knapp dreieinhalb Stunden Sitzungszeit und (wie üblich) ohne kontroverse Diskussion verabschiedet. Interessanterweise waren bei einigen Abgeordneten Unsicherheiten über die Konsequenzen des Referendums festzustellen. Mehr als einmal machten Kabinettsminister deutlich, daB die MCP nicht abgewählt wurde. Ein Redebeitrag wurde deshalb sogar durch Intervention eines Ministers unterbrochen und den restlichen Abgeordneten empfohlen, die Gesetzesvorlage "im Stillen" zu unterstützen.47 Ansonsten wurde Banda für die Durchfohrung des Referendums und Akzeptanz des Resultats gedankt. Natülich versäumten es die Abgeordneten auch nicht, die "glorreichen historischen Taten" des Staatsoberhauptes zu loben (wie sie es schon fast 30 Jahre zu tun pflegen!).

Zu der historischen Parlamentssitzung waren zahlreiche regimekritische einheimische Journalisten angereist, die aber, obwohl vorher genehmigt, auf Beschluß des Parlamentspräsidenten wieder ausgeschlossen wurden. Nur zwei couragierten Zeitungsleuten gelang es, ihre Anwesenheit lautstark durchzusetzen 48 - immerhin ein Zeichen für die Offnung des Systems.

Das Ergebnis des Referendums wurde sogar innerhalb der MCP unterschiedlich aufgenommen. Während sich einige hohe, aber besonders loyale, Parteifunktionäre völlig schockiert über ihre Niederlage zeigten 49 , war sie von anderen, die zum inneren Zirkel der Macht zähl(t)en, erwartet worden. Ein ehemaliger Minister und hoher Funktionär erklärte Banda schon Ende April 1993 seinen Rücktritt, der zwar angenommen, aber erst im Juli 1993 veröffentlicht wurde.50 Er war damit - neben dem ehemaligen Justizminister F. Makuta - das zweite Kabinettsmitglied, das auf eigenen Wunsch ausschied, um anscheinend noch rechtzeitig den Absprung zu schaffen. Noch vor kurzer Zeit wäre eine Demission als Respektlosigkeit gegenüber dem Präsidenten geahndet worden. Ein noch aktives Führungsmitglied der MCP sah das Debakel seiner Partei voraus und rechnet ihr auch für

45 Interview mit dem Of fizier C, 02.07.1993.

46 Hansard (Zomba), 29. Session, 4. Meeting, 29.06.1993.

47 Woran sich einige Abgeondnete aber nicht hielten. Vgl. Hansard, 29.06.1993.

48 Gespräch mit dem Joumalisten D am 03.07.1993.

49 So z.B. der Minister E (Interview 01.07,1993) und ein weibliches Mitglied des Exekutivkomitees der MCP, die besonders enttäuscht darüber war, da "ihre Frauen" zwar für Banda getanzt, ihn dann aber nicht unterstütz hätten (Interview, 24.06.1993).

50 Interview mit B am 21.06.1993. 
die geplanten Parlamentswahlen schlechte Chancen aus. Für das schlechte Abschneiden der MCP wird intern auch Selbstkritik laut: Das schlechte Image des Staatsministers (und "starken Mannes" hinter Banda), John Tembo, der in der Bevölkerung für zahlreiche Repressalien und Menschenrechtsverletzungen verantwortlich gemacht wird; Zwangsspenden an Banda während seiner jährlichen landesweiten Tourneen; Zwang zum Kauf von Parteiausweisen (sogar für Babies mußten welche erstanden werden) und Zwangsrekrutierung von Tänzerinnen für Bandas zahlreiche offentliche Aufuritte brachten die MCP bei der Bevölkerung in Mißkredit.51

Die durch das Referendum eingeleitete demokratische Transition wurde durch eine institutionalisierte Dialogplattform zwischen Regienung und Opposition vorangetrieben. Im "National Consultative Council" wird das weitere Vorgehen in der Ubergangsphase bis zu den Wahlen, die für Mai 1994 geplant sind, beraten. De jure ist dennoch nur die MCP an der Regierung.

Der wirkliche Verhandlungsproze $B$ findet zwischen den hohen Funktionären des Regimes und den Oppositionsführem hinter verschlossenen Türen statt. Die staatlichen Institutionen, wie etwa das Parlament, dienen nur als formale Instanz, die die Verhandlungsergebnisse in Gesetze umsetzt. In der Regierung sind "Hardliner" (um John Tembo) und "Gemäßigte" zu unterscheiden. Das gleiche gilt für die zwei wichtigsten Oppositionsparteien: In der "Aford" gibt es starke Kräfte um den - nicht unumstrittenen - Führer Chihana, die fast jede Kompromißbereitschaft gegenüber dem Regime ablehnen und sogar die UDF-Führer, die fast alle geschaßte ehemalige MCP-Politiker waren, für ihre damaligen Vergehen juristisch zur Rechenschaft gezogen sehen wollen. Als weitaus pragmatischer und kompromißbereiter zeigt sich die UDF. Hierin wird deutlich, daß die Kooperationsmöglichkeiten der größten Oppositionsparteien untereinander begrenzt sind. 52

Gerade darin besteht die Chance der MCP, die Wahlen gewinnen zu können. Wie das Beispiel Kenya lehrt, hat eine zersplitterte Opposition kaum Chancen, das alte Regime abzulösen. Eine solche Entwicklung sollte durch die Generalamnesty für Exilpolitiker offensichtlich begünstigt werden. Bisher konnten die ehemaligen Exilparteien noch keine zahlenmäßig signifikante Anhängerschaft reknutieren.

Viel wird auch vom Wahlsystem abhängen. Während das Verhältniswahlrecht die kleineren Parteien begünstigt, würde die britische Mehrheitswahl einen Großteil der Wählerstimmen für die Sitzverteilung im Parlament unberücksichtigt lassen. Sollte für die Wahl eines

51 Interview mit A, 23.06.1993. Genau diese Kritikpunkte wurden (neben fallendem Lebensstandard) auch in den vom Autor besuchten Dörfem in der Südregion genannt.

52 Funktionäre beider Parteien äußerten sich dem Autor gegenüber optimistisch, jeweils alleine dic absolute Mehmeit im Parlament zu gewinnen, was kaum mehr als eine Ilusion sein dürfte. 
Exekutivpräsidenten die relative Mehrheit ausreichen (wie in Kenya), hätte - ethnisch orientiertes Wahlverhalten unterstellt - Chihana, der den dünn besiedelten Norden repräsentiert, geringe Chancen. Das Rennen dürfte zwischen der UDF und MCP gemacht werden, wobei ausschlaggebend sein wird, welche Persönlichkeit antritt. In der UDF ist bisher noch kein herausragender Kandidat auszumachen. Die MCP ist durch Banda paralysiert, dem wohl das Recht auf die Kandidatur zustehen dürfte, der aber wegen seines hohen Alters (über 90 Jahre) und seines angegriffenen Gesundheitszustandes kaum eine Perspektive bietet. Sollte Banda gewählt werden, wäre die Nachfolgefrage nicht von der Tagesordnung. Die besten Chancen hätte die MCP vermutlich mit einem reformfreudigen, kompromißbereiten Kandidaten.

Für die Übergangsphase bis zu den Wahlen symbolisiert Banda eine nicht zu unterschätzende politische Stabilität. Das wurde auch der Opposition deutlich, als der Präsident wegen einer schweren Erkrankung die Amtsgeschäfte vorrubergehend nicht mehr führen konnte (Oktober 1993). Sie reagierte überrascht und hilflos. Weder die UDF noch die Aford nutzten die (zu diesem Zeitpunkt vermutlich günstige) Gelegenheit, ihre feste Einbindung in eine Übergangsregienung zu erreichen. Obwohl beiden Parteien offenbar von der MCP eine Repräsentation in einem "Presidential Council" angeboten wurde, reagierten sie unentschlossen. Das ist ein Zeichen für die (noch) geringe Professionalität der Opposition. Beide Parteien haben mit internen Problemen zu kämpfen. So sind mehrere UDFFunktionäre zur MCP übergelaufen, unter anderem der erste stellvertretende Vorsitzende Chaziya, der sich von der MCP mit dem Botschaftersessel in London ködern ließ.53 In der Aford, die organisatorisch schwach ist und politisch ein noch immer eher diffuses Bild abgibt, ist es anscheinend zu verdeckten Flügelkämpfen gekommen, von denen wohl auch die Autorität Chihanas nicht unangetastet bleiben wird. 54

Beide wichtige Oppositionsparteien konnten sich die Führungsschwäche des Regimes bisher nicht zu Nutze machen, um eine aktivere Rolle im demokratischen Transitionsprozeß zu spielen.

Das Ausfallen Bandas wurde durch die (für solche Fälle verf assungsmäBig vorgesehene 55 ) Bildung eines Präsidialrates (Presidential Council), der die Aufgaben des Präsidenten bis zu seiner erneuten Amtsfähigkeit oder bis zur Wahl eines Nachfolgers wahmimmt, überbrückt. Die personelle Zusammensetzung des Rates ist interessant: Den formalen Vorsitz führt der Generalsekretär der MCP, eine Position, die fast eine Dekade vakant blieb und aus rein taktischen Gründen mit dem erst Mitte 1993 nach einer 13-jährigen Haft aus dem Gefängnis entlassenen Chakuamba Phiri besetzt wurde. Chakuamba wurde von der UDF abgewor-

53 Africa Confidential, 34, 3, 22.10.1993, S. 6f.

54 Africa Confidential, 22.10.1993, S. 7.

55 Constitution of Malawi, Chapter II, 13 (1)-(2). 
ben. Er war ein hoher und einflußreicher MCP-Politiker und wurde wegen eines angeblichen Attentatsversuchs auf Präsident Banda wegen Hochverrats verurteilt.56 Er hat offenbar kaum mehr als eine Symbolfunktion und soll zum besseren Image der MCP beitragen. Der Drahtzieher hinter den Kulissen ist der im Volk unbeliebte Staatsminister John Tembo, der als zweites Mitglied des Rates als politisches Schwergewicht zu bezeichnen ist. Das dritte und letzte Mitglied Robson Chirwa gilt als Ergebener Tembos ohne eigenes politisches Profil. Die Zusammensetzung des Präsidialrates kam somit einer de facto - Machtübernahme Tembos gleich57. Dieser für die Transition ungünstigen Entwicklung hatte die Opposition offensichtlich nichts entgegenzusetzen.

Für den weiteren Weg der Demokratisienung, die durch das Referendum eingeleitet wurde, sind verschiedene Faktoren wichtig:

- Wird es der Opposition gelingen, in den Wahlen zu kooperieren, um das Regime abzuwählen; oder kommt es zu einer (nicht auszuschließenden) Kooperation von MCP und UDF nach den Wahlen?

- werden es die Oppositionsparteien schaffen, eine landesweite Organisationsstruktur aufzubauen und eine programmatisch - nicht ethnisch - orientierte Anhängerschaft zu rekrutieren?

- welche Rolle wird das Militär spielen? Wird es seine bisherige politische Neutralität bewahren oder in bestimmten Konstellationen in das Geschehen eingreifen?

Die Zerstrittenheit der Oppositionsparteien intern und zueinander erhöht die Chancen der MCP, durch Zersplitterung der Oppositionsstimmen die Wahlen zu gewinnen. Die ethnische Polarisierung der Parteien ist bis zu den Wahlen kaum zu entschärfen. Während die Aford eher demokratischen Ideen verpflichtet zu sein scheint, gibt sich die UDF pragmatisch. Ihre Funktionäre verfügen über Politikerfahrung aus den Zeiten ihrer MCP-Tätigkeit. Mit ihren MCP-Kollegen verbinden sie eine schwere Hypothek: Viele von ihnen waren als Regime-Mitglieder an Repressionen und vielleicht auch Komption beteiligt. Ginge es nach Chihana und der Aford, müßten alle Sünden der Vergangenheit gerichtlich geklärt und geahndet werden58. Das wäre zwar wünschenswert, aber mit der UDF kaum zu machen. Viele UDF-Politiker waren vom Zugriff auf staatliche Ressourcen bisher ausgeschlossen (nachdem sie bei Banda in Ungnade gefallen waren).

Ob die demokratische Transition nur Auswirkungen auf die dünne urbane Bevölkerung hat und kaum mehr als eine Art "Elitenzirkulation" sein wird, bleibt abzuwarten.59

56 Vgl.: Süddeutsche Zeitung, 06./07.11.1993.

57 G. Mhone nannte es ein "quiet palace coup d'état", in: Southem Africa Political \& Economic Monthly (Harare), 7, 2, November 1993, S. 21-23.

58 Interview mit dem Aford-Funktionären E und F, 28.06.1993.

59 Der Autor arbeitet an einem empirischen Forschungsvorhaben zur demokratischen Transition in Malawi. 
Das Militär hat sich Ende 1993 erstmals in die Innenpolitik eingemischt, indem es verschiedene Basen und Einrichtungen (danmter off enbar auch das Hauptquartier) der "Jungen Pioniere" (MYP) verwüstete und Mitglieder der paramilitärischen Organisation, die als Macht- und Unterdrückungsinstrument Bandas diente und von den Streitkräften als "Konkurrenzarmee" bemißäugt wurden, umbrachte. 60

Dieses Vorgehen läßt sich als Wamung an Tembo deuten, den demokratischen Transitionsprozeß nicht abzubrechen. Tatsächlich beschloß der Präsidialrat nach den Zwischenfällen, die "Jungen Pioniere" zu entwaffnen.61

Entgegen allen Vermutungen hat Präsident Banda Anfang Dezember 1993 seine Amtsgeschäfte wieder aufgenommen62. Der greise Startschef genießt auch in der Opposition einen gewissen Respekt und könnte sein Bild in der Geschichte als Diktator durch eine friedliche Demokratisierung korrigieren. Welche Konsolidierungschancen ein pluralistisches System in einem armen Agrarstaat wie Malawi letztendlich haben wird, steht auf einem anderen Blatt. Auch ein Wahlsieg der MCP wirde vermutlich nicht das Ende der Demokratisierung bedeuten, denn die gewonnene Freiheit (Menschen- und Bürgerrechte, legale politische Opposition, freie Presse etc.) läßt sich so einf ach nicht in die einmal geöffnete "Büchse der Pandora" zurücksperren.

60 Neue Zürcher Zeitung, 05.12.1993; Svenska Dagbladet, 05.12.1993.

61 Neue Zürcher Zeitung, 05.12.1993.

62 Intemational Herald Tribune, 06.12.1993. 
disclosure and the denunciation of human rights violations rather than on coercive measures. In its thematic part, the Declaration pays particular attention to persons belonging to disadvantaged groups. On the whole, the Declaration is not likely to become a milestone in the development of international law.

\section{Malawi on the Way to Democracy? The Referendum and its Consequences}

\section{By Heiko Meinhardt}

Under growing international and domestic pressure on the Malawi government to carry out democratic reforms President Hastings Banda called for a National Referendum which was held on 14th June, 1993. It was the first opportunity for the Malawians to participate in a free and fair poll. Almost two thirds of the voters voted for the introduction of multipartyism and against the Banda regime which rules the country since its independence in 1964.

The referendum was the starting point for the process of democratic transition in Malawi. However, the bargaining process between the two major opposition parties and the current regime is dominated and to some extent clearly controlled by the latter. For a success of the opposition in the forthcoming general elections (scheduled for May 1994) it will be important for the newly established parties to organize themselves nationwide on the basis of a political program and to avoid the predominance of a certain ethnic group. Their success seems unlikely without some cooperation because the government could make the game easily against a weak, fragmented and quarreled opposition. However, the chances for such an improvement seem not very high. Although a victory of the ruling party would not seem to be the best alternative it does not necessarily mean an end of the democratisation process since civil and human liberties were granted. 